\title{
Effect of Serum Concentration on Hypothermic Preservation of Cardiac Myocytes Isolated from Neonatal Rat Ventricle
}

\author{
Hiroyuki Orita, M.D., Manabu Fukasawa, M.D., \\ Shigeki Hirooka, M.D., Hideaki Uchino, M.D., \\ Kana Fukui, M.D., Minoru KoHI, M.D., \\ Masahiko Washio, M.D., \\ and Takahiko Suzuki, Ph.D.*
}

\section{SUMMARY}

The purpose of the present study was to evaluate the functional and biochemical effects of serum concentrations on hypothermic preservation of cardiac myocytes. Myocytes were isolated from neonatal rat ventricles and cultured for 4 days, and then incubated for $24 \mathrm{hrs}$ at $4^{\circ} \mathrm{C}$ in media containing $0,2,5,10$, and $20 \%$ fetal calf serum (FCS). The myocytes were then cultured for an additional $24 \mathrm{hrs}$ at $37^{\circ} \mathrm{C}$ to evaluate the recovery of myocyte beating rate.

The recovery ratio of myocyte beating rate after hypothermic incubation was $25.3 \pm 14.2$ percent of control in the serum-free, $33.0 \%$ in $2 \%$ FCS, $30.7 \%$ in $5 \% \mathrm{FCS}, 22.3 \%$ in $10 \% \mathrm{FCS}$, and $24.3 \%$ in $20 \% \mathrm{FCS}$ groups. Serum-free, $10 \%$ and $20 \%$ FCS groups had lower recovery ratios compared to the other two groups. The release of CPK and LDH after hypothermic incubation were similer in the serum-free, $2 \%$ and $5 \%$ FCS groups, however, significant increases were observed for the $10 \%$ and $20 \%$ FCS groups.

It is suggested that fetal calf serum at high concentrations $(>10 \%)$ might be injurious to immature myocardium under hypothermic preservation, and that the optimum concentration of FCS is two percent or less. (Jpn Heart J 35: 213-223, 1994)

Key words: Immature myocyte Myocyte culture Hypothermic injury Fetal calf serum

\footnotetext{
YARDIAC transplantation is now a successful treatment for irreversible, final cardiac failure." However, problems still remain, in particular, the relatively short time limit for myocardial preservation during explantation and stor-

From the Second Department of Surgery, Yamagata University School of Medicine and *Bioscience Laboratory Inc., Yamagata, Japan.

This work was supported by a grant from the Ministry of Education, Culture and Science, Japan.

Address for correspondence: Hiroyuki Orita, M.D., The Second Department of Surgery, Yamagata University School of Medicine, Iida-nishi, Yamagata, 990-23, Japan.

Received for publication September 1, 1993.

Accepted January 7, 1994.
} 
age. Recently, the donor heart in the transplantation has been preserved under hypothermic and aerobic conditions in an attempt to prolong the preservation time. ${ }^{2.37}$ Moreover, although several groups have investigated the long-term preservation of adult myocardium, ${ }^{2-6)}$ there have been few reports on immature myocardium. ${ }^{7-9)}$ Therefore, protection against hypothermic cellular injury of immature myocytes may become a future concern. The purpose of the present study was to evaluate the functional and biochemical effects of serum concentrations on hypothermic preservation of cardiac myocytes isolated from neonatal rat ventricle.

\section{Materials and Method}

Isolation of cardiac myocytes: Cardiac myocytes were prepared from neonatal rat ventricles by a modification of Klein's method. ${ }^{10,11)}$ Hearts were removed from 1-2 day-old neonatal Wistar male rats after decapitation. The ventricles from 20 hearts were minced into fine fragments with scissors in $0.025 \mathrm{M}$ Hepes buffered minimum salt solution (MSS, Gibco, Grand Island, NY). The fragments were rinsed twice with MSS to remove contaminating red blood cells and placed in a $50 \mathrm{~m} l$ flask containing $10 \mathrm{~m} l$ of $0.1 \%$ collagenase (Wako Chemical, Tokyo) in MSS. The flask was gently agitated for 60 minutes at $37^{\circ} \mathrm{C}$. The enzyme digest was centrifuged at $1000 \times \mathrm{g}$ for 2 minutes and the pellet washed twice with MSS. The resuspended cells and small aggregates were gently passed through a wiremesh screen ( $90 \mu \mathrm{m}$ porosity) to remove large aggregates and debris. The cellular filtrate was suspended in MCDB (Department of Molecular, Cellular and Developmental Biology, Colorado Univ.) 107 medium (Kyokuto Pharmaceutical, Tokyo) containing 5\% fetal calf serum (FCS) (Flow Labs, Rockville, MD) and placed into $75 \mathrm{~cm}^{2}$ tissue culture flasks which were incubated for 60 minutes at $37^{\circ} \mathrm{C}$ in a humidified atmosphere of $5 \% \mathrm{CO}_{2}: 95 \%$ air in order to separate the pure cardiac myocytes from the fibroblasts by a differential adherence protocol. $^{(1-14)}$ After incubation, unattached cells were decanted, centrifuged, and rinsed twice with MSS. Thereafter, the cell pellet was resuspended in MCDB 107 containing $2 \%$ FCS, transferrin $(10 \mu \mathrm{g} / \mathrm{ml}$, Sigma, St. Louis, MO), and insulin $(10 \mu \mathrm{g} / \mathrm{m} l \text {, Sigma })^{11)}$ and then gently passed through a $28 \mu \mathrm{m}$ wire-mesh screen (>95\% myocytes). ${ }^{11-16)}$ Myocytes were identified by their beating activity which began on the lst day of culture.

Gardiac myocyte preparation in culture flasks: Cardiac myocytes were cultured in $25 \mathrm{~cm}^{2}$ flasks which had been previously treated with fibronectin (Wako Chemical, Tokyo). ${ }^{1 /}$ Fibronectin treatment was performed by adding $1 \mathrm{ml}$ of 10 $\mu \mathrm{g} / \mathrm{ml}$ fibronectin in phosphate-buffer-saline (PBS), pH 7.4, to each culture flask and incubating at room temperature for 1 hour just prior to the addition of 
myocytes. The unbound fibronectin was decanted before the addition of myocytes. Thereafter, cell cultures were maintained at $37^{\circ} \mathrm{C}$ in a humidified atmosphere of $5 \% \mathrm{CO}_{2}: 95 \%$ air and the media was changed daily. A myocyte concentration of $2.5 \times 10^{5}$ cells $/ \mathrm{m} l$ was chosen and the total number of cells $/$ flask was $1.5 \times 10^{6}$ cells. ${ }^{15,16)}$

Cardiac myocyte hypothermic incubation at varying serum concentrations: On the 4th day of culture when the myocyte beating rate became stable, ${ }^{16}$ the myocytes were incubated for 24 hours at $4^{\circ} \mathrm{C}$ in media containing $0,2,5,10$, and $20 \%$ FCS which was heated at $56^{\circ} \mathrm{C}$ for 30 minutes (heat inactivation) (osmolarity: $301.4 \pm 3.1 \mathrm{mOsm}$; range 297.0 to 306.7 ). After each hypothermic incubation, the media was changed again and the myocytes were incubated at $37^{\circ} \mathrm{C}$ for an additional 24 hours in a humidified atmosphere. Triplicate flasks were evaluated for each group and the experiment was repeated an additional five times. The investigation was performed in accordance with the "Guidance on the Operation of the Animals (Scientific Procedures) Act 1986," published by Her Majesty's Stationary Office, London.

Cardiac myocyte beating rate recovery after hypothermic incubation: The contraction rate of each flask was observed and the mean contraction or beating rate of 10 myocytes/culture flask was determined microscopically. The myocyte beating rate was observed before and 24 hours after hypothermic incubation, and the recovery ratio of the myocyte beating rate was expressed as a percent of control (ie, beating rate prior to hypothermic incubation). Prior to hypothermic incubation, most of the cultured cells $(>95 \%)$ beat steadily and synchronously.

CPK and $L D H$ release from cardiac myocytes after hypothermic incubation: After each hypothermic incubation, creatine phosphokinase (CPK) and lactate dehydrogenase $(\mathrm{LDH})$ were measured in the storage solutions by using CPK or LDH assay kits (Unikitrate CPK or LDH, Chugai Pharmaceutical Co., Ltd., Tokyo) and a spectrophotometer (RaBA 3030, Chugai Pharmaceutical Co., Ltd., Tokyo) (L1). As a control, the storagc solutions without cultured cells were incubated for 24 hours at $4^{\circ} \mathrm{C}$, and then CPK and LDH levels were measured (L2). Thus, the values of CPK and $\mathrm{LDH}$ released from culturcd cells were determined by the following calculation and were expressed in international units (IU) per culture flask.

\section{Released CPK and LDH levels=L1-L2}

In addition, neither enzymal release from cultured cells increased significantly 24 hours after normotherrnic incubation at $37^{\circ} \mathrm{C}$.

Mortality of cardiac myocytes after hypothermic incubation: Twentyfour hours after hypothermic incubation, the mortality of cardiac myocytes was evaluated by the trypan-blue exclusion test. The mortality ratio was determined 
by evaluating 200 myocytes from photographs of each group.

Statistical analysis: Data were initially evaluated by rankit analysis to determine the distribution and then by Kruskal-Wallis one-way analysis of variance to examine the differences within the group, followed by the Mann-Whitney $U$ test. Results are presented as a meantstandard deviation and differences were considered significant if the $\mathrm{p}$ value was less than 0.05 .

\section{Results}

Cardiac myocyte beating rate recovery after hypothermic incubation: The average myocyte beating rate just prior to hypothermic incubation for all groups was $262.2 \pm 32.8$ beats $/ \mathrm{min}$ (range 241.2 \pm 30.4 to $269.0 \pm 27.7$ ). There were no significant differences among the groups. The recovery ratios of the myocyte beating rate after 24 hours of hypothermic incubation at $4^{\circ} \mathrm{C}$ in media containing various serum concentrations were $25.3 \pm 14.2$ percent of control in the serumfree group, $33.0 \pm 14.9$ in the $2 \%$ FCS group, $30.7 \pm 10.3$ in the $5 \%$ FCS group, $22.3 \pm 17.6$ in the $10 \%$ FCS group, and $24.3 \pm 13.1$ in the $20 \%$ FCS group. There were no significant differences among the five groups, however, the serum-free, $10 \%$ and $20 \%$ FCS groups had lower recovery ratios than to the other two groups (Figure 1).

CPK and LDH release from cardiac myocytes after hypothermic incubation: Prior to changing from culture media to hypothermic storage solu-

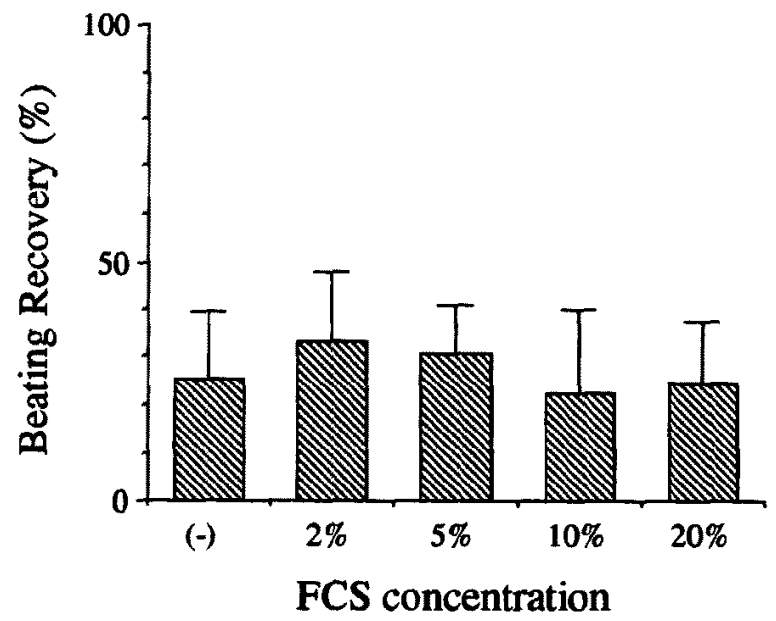

Figure 1. Recovery ratio of myocyte beating rate measured 24 hours after hypothermic prescrvation $\left(4^{\circ} \mathrm{C}\right)$ for 24 hours in media containing $0,2,5,10$, and $20 \%$ fetal calf serum (FCS). A total of $1.5 \times 10^{6}$ myocytes/culture flask were used, and each value is expressed as a percent of control (beating rate prior to hypothermic incubation) and represents the meantstandard deviation (SD) of six experiments. 


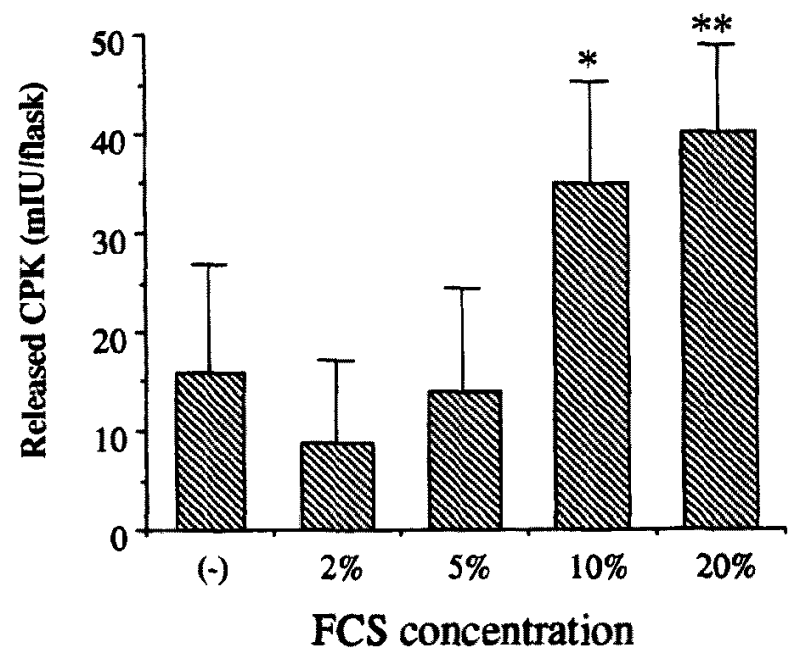

Figure 2. Released CPK levels in incubation medium containing various serum concentrations after 24 hours of hypothermic preservation. Each value represents the mean $+\mathrm{SD}$ of six experiments. *and **=significant differences from the $5 \%$ FCS group, $\mathrm{p}=0.025$ and 0.01 , respectively.

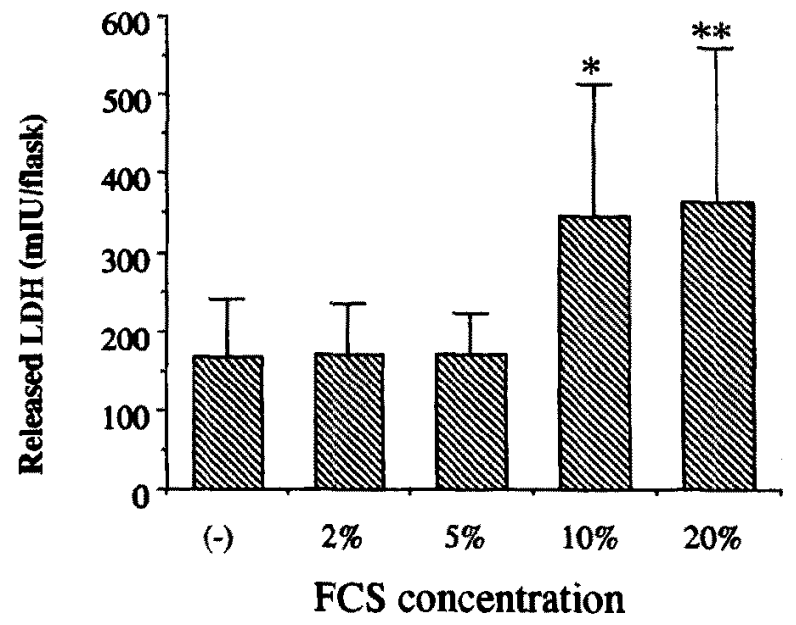

Figure 3. Released $\mathrm{LDH}$ levels in incubation medium containing various serum concentrations after 24 hours of hypothermic preservation. Each value represents the meantSD of six experiments. *and **=significant differences from the $5 \%$ FCS group, $\mathrm{p}=0.05$ and 0.01 , respectively.

tions, the culture media levels of CPK and LDH for the previous 24 hours were similar in all groups (CPK: $10.3 \pm 4.1 \mathrm{mIU} /$ flask, range $8.6 \pm 3.9$ to $11.8 \pm 6.7$; $\mathrm{LDH}: 172.6 \pm 23.1 \mathrm{mIU} /$ flask, range $151.6 \pm 19.2$ to $196.5 \pm 31.3)$. The releases of CPK after 24 hours of hypothermic incubation at $4^{\circ} \mathrm{C}$ in media containing various serum concentrations were similar for the serum-free, $2 \%$ and $5 \%$ FCS 


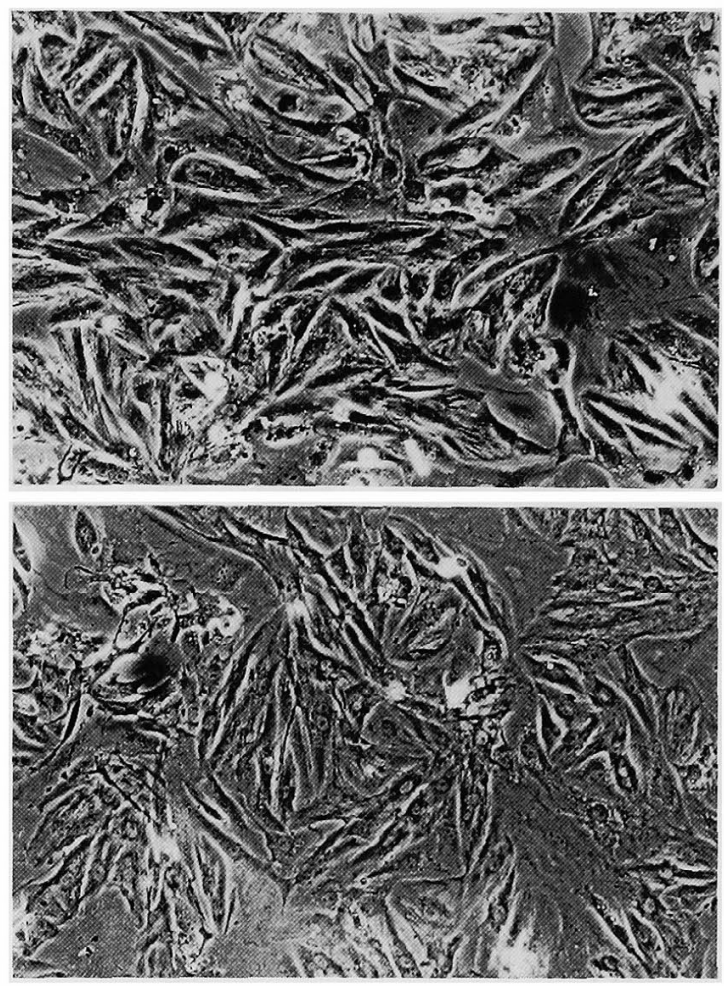

Figure 4. Photograph $(\times 50)$ of cardiac myocytes. Top: myocytes before hypothermic preservation; Bottom: myocytes at 24 hours after hypothermic preservation in media containing $2 \%$ fetal calf serum (FCS).

groups (15.9 $\pm 11.0,8.9 \pm 8.1,13.9 \pm 10.5 \mathrm{mIU} /$ flask, respectively), while significant increases in the $10 \%$ and $20 \%$ FCS groups were observed $(34.8 \pm 10.4 \mathrm{mIU} /$ flask, $\mathrm{p}<0.025$ vs the $5 \%$ FCS group; $40.0 \pm 8.8 \mathrm{mIU} /$ flask, $\mathrm{p}<0.01$ vs the $5 \%$ FCS group, respectively) (Figure 2).

The release of $\mathrm{LDH}$ after 24 hours of hypothermic incubation at $4^{\circ} \mathrm{C}$ in media containing various serum concentrations was similar to that for the serumfree, $2 \%$ and $5 \%$ FCS groups $(165.5 \pm 73.6,170.0 \pm 63.7,169 \pm 52.3 \mathrm{mIU} /$ flask, respectively), and increased significantly in the $10 \%$ and $20 \%$ FCS groups (346.1 $\pm 166.3 \mathrm{mIU} /$ flask, $\mathrm{p}<0.05$ vs the $5 \%$ FCS group; $362.8 \pm 193.5 \mathrm{mIU} /$ flask, $\mathrm{p}<0.01$ vs the $5 \%$ FCS group, respectively) (Figure 3 ).

Mortality of cardiac myocytes after hypothermic incubation: At 24 hours after hypothermic incubation, myocytes in the serum-free, $2 \%$ and $5 \%$ FCS groups showed minor morphological changes compared to the control myocytes (i.e., myocytes prior to hypothermic incubation, Figure 4) with mortality rates of $4.2 \pm 1.9,3.8 \pm 1.6,4.8 \pm 1.8$ percent, respectively, as determined by trypan-blue exclusion. In contrast, the $10 \%$ group myocytes showed major morphological 

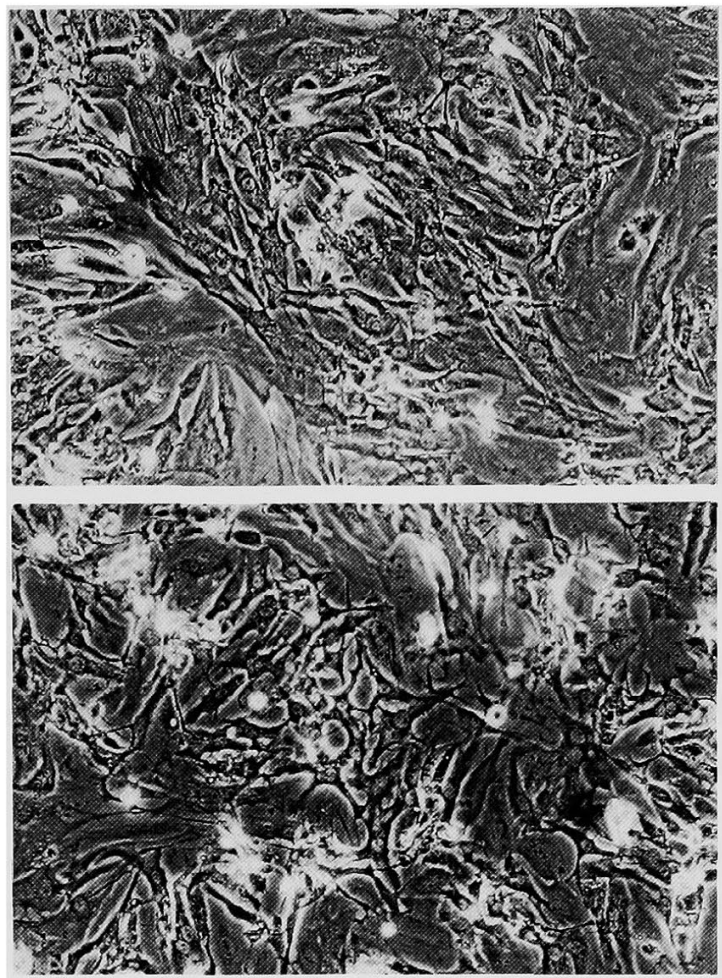

Figure 5. Photographs $(\times 50)$ of cardiac myocytes. Top: myocytes at 24 hours after hypothermic preservation in media containing 10\% FCS; Bottom: myocytes preserved in media containing $20 \%$ FCS.

changes which included cellular atrophy and peeling off the bottom of the culture flasks (Figure 5). The $10 \%$ group mortality rate was $8.3 \pm 3.7$ percent $(p<0.05$ vs the $5 \%$ group). Moreover, marked morphological changes were observed in the $20 \%$ group with a mortality rate of $9.2 \pm 4.1$ percent $(\mathrm{p}<0.025$ vs the $5 \%$ group) (Figure 5).

\section{Discussion}

Cardiac myocytes isolated from neonatal rat ventricles formed conflusnt monolayers by the fourth day of culture, at which time almost all (>95\%) beat synchronously and steadily at a constant frequency. They were maintained for five days of culture, after which the beating decreased gradually for 21 days. ${ }^{15,16)}$ The myocytes did not beat, or beat weakly, just after hypothermic incubation, following which they beat synchronously and steadily. The duration of the constant frequency of myocyte beating has been shown to differ under various conditions of hypothermic incubation, although almost all the myocytes showed 
synchronous and steady beating by 24 hours after hypothermic incubation. ${ }^{15,16}$ Accordingly, in this study, we used myocytes on the fourth day of culture and evaluated the myocyte beating rate recovery 24 hours after hypothermic incubation.

Myocardial hypothermia has been used routinely in open heart surgery, especially in neonates or during heart transplantation. Recently, the donor heart in heart transplantations has been preserved in hypothermic and aerobic conditions by perfusing oxygenated solution in order to elongate the preservation time. ${ }^{2,3)}$ Therefore, protection against hypothermic cellular injury may become an important problem. Previously, we investigated myocyte viability after hypothermic incubation in MCDB 107 medium containing $2 \%$ fetal calf serum (FCS) using cultured myocytes isolated from neonatal rat ventricles. The $4^{\circ} \mathrm{C}$ hypothermia significantly induced immature myocyte injury both functionally and biochemically, ${ }^{16)}$ although generally it has not been shown to be injurious to adult myocardium. ${ }^{5}$. Thus, in this study, we evaluated the effects of serum concentrations on hypothermic injury to immature cardiac myocytes.

Long-term hypothermic $\left(4^{\circ} \mathrm{C}\right)$ preservation using tissue culture medium containing FCS has been demonstrated by several investigators for the storage of aortic homografts. ${ }^{17-19)}$ Bodnar et al ${ }^{18)}$ used a medium containing $10 \%$ FCS in order to maintain tissue viability. In the present study, we examined the effects of serum concentrations on immature myocyte preservation. The decline in myocyte functional activity as measured by percent recovery of the myocyte beating rate 24 hours after hypothermic incubation was observed in serum-free, $10 \%$ and $20 \%$ FCS groups, although there were no significant differences among the groups. The evaluation of myocytes using beating rate recovery, as opposed to the more comprehensive contractility measurements, may not accurately reflect all parameters of myocyte function, but appears to have utility since myocytes respond rapidly to various environmental or chemical types of damage. ${ }^{15,16,20,21\rangle}$ In contrast, cellular release of the biochemical markers CPK and LDH increased significantly in the $10 \%$ and $20 \%$ FCS groups. These two cytoplasmic enzymes have been shown to increase linearly with culture time, ${ }^{16)}$ and the release into culture media is indicative of damage to cultured cells. ${ }^{14-16,22,23)}$ Therefore, high concentrations of the serum biochemically accelerated myocyte injury under hypothermic conditions. In addition, myocytes in the $10 \%$ and $20 \%$ FCS groups might show critical expression functionally, as observed by morphological findings which included cellular atrophy and peeling off the bottom of the culture flasks. Accordingly, fetal calf serum at the concentrations of $10 \%$ or greater may be injurious to the immature myocytes under severe hypothermic conditions. Moreover, FCS may not necessarily be an essential agent in order to maintain myocyte viability during hypothermic preservation, although the $2 \%$ 
FCS group myocytes exhibited the best recovery of the myocyte beating rate and the least release of biochemical markers.

High concentrations (above 10\%) of FCS exhibited an injurious effect on myocytes from hypothermic incubation. This effect was also observed in a different batch of FCS tested (SEBAK, Aidenbach, Germany). The precise mechanisms by which these phenomena occur are not well understood, however, high colloid osmolarity may be responsible for the myocyte injury in this study, while the osmolarity itself was similar among our five groups. Previously, several investigators have reported that hyperosmolar or colloid hyperosmolar storage solutions had protective effects on adult myocardium at $4^{\circ} \mathrm{C}$ preservation..$^{24,25)}$ Toledo-Pereyra et $\mathrm{al}^{24)}$ documented that canine hearts preserved under hypothermic storage for 24 hours with hyperosmolar colloid solution $(450 \mathrm{mOsm})$ had a significantly better response than did the hearts preserved for the same period of time with a crystalloid solution (Ringer's). They speculated that the hyperosmolarity would prevent cellular swelling and interstitial edema.

In contrast, in our study, no additional protective effects were observed by increasing colloid osmolarity. Hypothermia has been shown to increase free cytosolic calcium, derived from the sarcoplasmic reticulum, which in turn induces cardiac muscle contracture and increased cellular damage. ${ }^{26,27)}$ Hypothermia itself has been shown to induce direct damage to the sarcolemma. ${ }^{15,16)}$ In this report the injurious effects of high colloid osmolarity may be mediated by the decrease in sarcolemmal stability under hypothermic conditions. In addition, the injurious effects may be induced by cytotoxic substances in the FCS. Low density lipoprotein (LDL), which is not affected by heat inactivation, seems to be one serum component that inhibits sarcoplasmic metabolism. ${ }^{11)}$ However, the effects of cytotoxic substances appear to be less since the cellular release of two biochemical markers (CPK and $\mathrm{LDH}$ ) did not increase significantly 24 hours after normothermic incubation at high concentrations of FCS (range 10 to $20 \%$ ). ${ }^{11)}$ Thus, it is speculated that serum at high concentrations may be unsuitable for hypothermic preservation of immature myocardium, which might have different characteristics compared to adult myocardium under hypothermic conditions. Further investigations will be necessary in order to evaluate the effects of hyperosmolar storage solutions on immature myocytes under hypothermic conditions.

The in vitro cell-culture system reported here has the following advantages for evaluating hypothermic preservation: (a) ease of observation and measurement of biochemical response to treatments such as storage solutions or cytoprotective agents; (b) maintenance of a well-defined biochemical and physical environment; and (c) the ability to cvaluate multiple treatments in a single experiment. Furthermore, this culture system provides a model for evaluating direct 
cellular injury under ischemic or postischemic reperfused conditions. However, the results from this in vitro model are not equal to those of the in vivo condition. Thus, further in vivo examinations will be needed in order to confirm these results.

In summary, immature myocyte injury, both functionally and biochemically, was accelerated by hypothermic incubation in media containing $10 \%$ and $20 \%$ fetal calf serum. Therefore, it is suggested that fetal calf serum at high concentrations may be injurious to immature myocardium during hypothermic preservation, and that the optimum concentration of FCS is two percent or less.

\section{ACKNOWLedGement}

The authors wish to thank Mr. Joseph D. Campeau for his assistance with the preparation of this manuscript.

\section{REFERENCES}

1. Heck CF, Shumway SJ, Kaye MP: The registry of the International Society for Heart Transplantation; sixth official report-1989. J Heart Transplant 8: 271, 1989

2. Burt JM, Copeland JG: Myocardial function after preservation for 24 hours. J Thorac Cardiovasc Surg 92: 238, 1986

3. Takahashi A, Braimbridge MV, Hearse DJ, Chambers DJ: Long-term preservation of the mammalian myocardium; effect of storage medium and temperature on the vulnerability to tissue injury. $J$ Thorac Cardiovasc Surg 102: 235, 1991

4. Wicomb W, Cooper DKC, Hassoulas J, Rose AG, Barnard CN: Orthotopic transplantation of the baboon heart after 20 to 24 hours' preservation by continuous hypothermic perfusion with an oxygenated hyperosmolar solution. J Thorac Cardiovasc Surg 83: 133, 1982

5. Warner M, Guerraty A, Alivizatos P, Choi SC, Hudson B, Lower RR, Hess ML: Assessment of myocardial subcellular function after 24 hours of in vitro preservation and transplantation. $J$ Thorac Cardiovasc Surg 83: 290, 1982

6. Galinanes M, Chambers DJ, Hearse DJ: Effect of sodium aspartate on the recovery of the rat heart from long-term hypothermic storage. J Thorac Cardiovasc Surg 103: 521, 1992

7. Jarmakani JM, Nakazawa M, Nagatomo $T$, Langer GA: Effect of hypoxia on mechanical function in the neonatal mammalian heart. Am J Physiol 235: H469, 1978

8. Bove EL, Gallagher KP, Drake DH, Lynch MJ, Fox M, Forder J, Boling SF, Shlafer M: The effect of hypothermic ischemia on recovery of left ventricular function and preload reserve in the neonatal heart. J Thorac Cardiovase Surg 95: 814, 1988

9. Grice WN, Konishi T, Apstein CS: Resistance of neonatal myocardium to injury during normothermic and hypothermic ischemic arrest and reperfusion. Circulation 76 (Suppl V): V-150, 1987

10. Klein I, Daood M: Regulation of the growth of nonmuscle heart cells in culture. In Vitro Cell Dev Biol 21: 693,1985

11. Suzuki T, Ohta M, Hoshi H: Serum-free, chemically defined medium to evaluate the direct effects of growth factors and inhibitors on proliferation and function of neonatal rat cardiac muscle cells in culture. In Vitro Cell Dev Biol 25: 601,1989

12. Blondel B, Roijen I, Cheneval JP: Heart cells in culture; a simple method for increasing the population of myoblasts. Experientia 27: 356, 1971

13. Polinger IS: Separation of cell types in embryonic heart cell culture. Exp Cell Res 63: 78, 1970

14. Yagev $S$, Heller M, Pinson A: Change in cytoplasmic and lysosomal enzyme activities in cultured rat heart cells; the relationship to cell differentiation and cell population in culture. In Vitro 20: 893, 1984 
15. Orita H, Fukasawa M, Hirooka S, Fukui K, Kohi M, Washio M, Sasaki H: Protection of cardiac myocytes from hypothermic injury by cardiac fibroblasts isolated from neonatal rat ventricle. $J$ Surg Res 55: 654,1993

16. Orita H, Fukasawa M, Hirooka S, Fukui K, Kohi M, Washio M: Cardiac myocyte culture system as an in vitro experimental model for evaluation of hypothermic preservation. Surgery Today 23: 439, 1993

17. Al Janabi N, Ross DN: Enhanced viability of fresh aortic homograft stored in nutrient medium. Cardiovase Res 7: 817, 1973

18. Bodnar E, Matsuki O, Parker R, Ross DN: Viable and nonviable aortic homografts in the subcoronary position; a comparative study. Ann Thorac Surg 47: 799, 1989

19. O'Brien ME, McGiffin DC, Stafford EG, Gardner MA, Pohler PF, McLachlan GJ, Gall K, Smith S, Murphy E: Allograft aortic valve replacement; long-tern comparative clinical analysis of the viable cryopreserved and antibiotic 4 degree C stored valves. J Card Surg 6:534, 1991

20. Orita H, Fukasawa M, Hirooka $\mathrm{S}$, Uchino H, Fukui $\mathrm{K}$, Kohi M, Washio M: In vitro evaluation of diltiazem on hypothermic injury to immature myocytes. Cardiovasc Drugs and Ther 7: 713, 1993

21. Hendry PJ, Labow RS, Barry TA, Keon WJ: An assessment of crystalloid solutions for donor heart preservation. J Thorac Cardiovasc Surg 101: 833, 1991

22. Van der Laarse A, Hollaar L, Kokshoorn LJM, Witteveen SAGJ: The activity of cardio-specific isoenzymes of creatine phosphokinase and lactate dehydrogenase in monolayer cultures of neonatal rat heart cells. J Mol Cell Cardiol 11: 501, 1979

23. Acosta D, Puckett M, McMillin R: Ischemic myocardial injury in cultured heart cells; Leakage of cytoplasmic enzymes from injured cells. In Vitro 14: 728, 1978

24. Toledo-Pereyra LH, Sharp HL, Condie RM, Chee M, lillehei RG, Najarian JS: Preservation of canine hearts after warm ischemia (zero to thirty minutes) and one to two days of hypothermic storage; a comparative analysis of crystalloid and colloid solutions with different osmolarity and ion composition. J Thorac Cardiovasc Surg 74: 594, 1977

25. Foglia RP, Steed DL, Follettc DM, DeLand E, Buckberg GD: Iatrogenic myocardial edema with potassium cardioplegia. J Thorac Cardiovasc Surg 78: 217, 1979

26. Bers DM, Bridge JHB, Spizer KW: Intracellular Ca transients during rapid cooling contractures in guinea-pig ventricular myocytes. J Physiol 417: 537, 1989

27. Hryshko LV, Stiffel V, Bers DM: Rapid cooling contractures as an index of sarcoplasmic reticulum calcium content in rabbit ventricular myocytes. Am J Physiol 257: H1369, 1989 